International Journal of Engineering \& Technology, $7(2.25)(2018) 17-19$
International Journal of Engineering \& Technology
SPC
Website: www.sciencepubco.com/index.php/IJET
Research paper

\title{
Implementation Of Wearable Radiation Detector
}

\author{
A.Josephin Arockia Dhivya *, R . Chandrasekaran ${ }^{2}$, Josline Elsa Joseph $^{3}$, T.R.Thamizhvani ${ }^{4}$, S.K.Arun ${ }^{5}$, V.Manoj $^{6}$ \\ Department of Biomedical Engineering, Vels Institute of Science,Technology and \\ Advanced Studies, Pallavaram, Chennai-600117, India \\ *Corresponding author Email: a.dhivya.se@velsuniv.ac.in
}

\begin{abstract}
Wearable devices are always in race to support and assist the human healthcare. The wearable devices are commonly used to monitor the physiological parameters of the humans and transmitting to the gadgets. In this paper we proposed a novel idea of monitoring the radiation in the environment and alerting the users about the radiation and level of radiation in the environment. In this paper the implementation of a radiation detector based on a semiconductor photodiode. The awareness of the hazardous radiation should be created and the people should aware of the hazardous radiation in the environment. The arduino controller proposed in the prototype acts as the heart of the wearable device. The detected radiation is transmitted through Bluetooth zixbee module and viewed in smart phones.
\end{abstract}

Keywords: BPW-34-Photodiode;arduino-uno

\section{Introduction}

The emission or transmission of electromagnetic waves on through the space is a material includes: $\mathrm{x}$-rays, gamma radiation, microwaves, electromagnetic radiation etc... Radiation can cause May harmful effect on human body it can cause leukaemia, skin burn, etc...[1].Due to cosmic radiation many people are suffering from defects.The electromagnetic radiation such as infrared, ultraviolet etc has been emitted by the sun. The sun corona which is upper layer of sun's atmosphere. Corona at millions of degree heat is hot enough to emit $\mathrm{x}$-rays which may cause tissue or skin damage. Ionizing radiation is harmful to health. Geiger counter alone is not used to measure harmful radiation.[2].Semiconductor sensor is used to detect sensitivity.The Geiger-Muller tube is first device that used to measure the radioactivity.These Geiger counter components are too costly and not economical.If it manageable too,still we need more volts for their working.Still its less know about photodiode like BPM34 also can detect radiation. Due to its small size it can be used as a wearable device.

\section{Literature Survey}

Dong Xue, et al... Designed a prototype of power-efficient antennas, Low-profile which are essential for

body-worn devices applicable for long-term healthcare monitoring. The fabrication of fold cylindrical helix $(\mathrm{FCH})$ antennas, and measurement of their performance on the human body is mainly focused in this paper. The study also includes one element and two element FCH arrays. The fabricated FCH can excite the same on-body propagation within a compact size, it has been found. Its on-body radiation performance is improved over the single $\mathrm{FCH}$ and the simulated two-element $\mathrm{FCH}$ array can achieve a higher gain.

Kei kamada, et al... Based on time over threshold method is developed, they developed A novel digital Pet scanner. To determine about large counting rate and space resolution, the positron emission Tomograph (PET) is composed of 144channel ce:Gd3Al2Ga3012 (GAGG) - avalanche photodiode(APC) counter arrays individually paired with time over threshold specific application integrated circuit. It comprises of a electrical circuit with a pulse generating system.A detector component will be present to measure the time.The measured energy will be nearly $70 \%$ for 525 peak and 4 seconds. Pawel olko, et al.. With different Li compositions (Li-6 and Li-7) they developed fluoride thermo luminescence and different components like Titanium,Magnesium,Copper are present.Probably they are used in dosimetry purposes. There is a layer known as Primary radiation in space, which has fast moving electrons,protons and heavily charged particles.Using this calculation of dosage efficiency are estimated.

D.T. Williams, et al...His study reveals about a low noise amplifier which comprises of a cadmium -sulfate crystal which is mounted in a hypodermic needle.It is mainly helpful to get the yielding rate in order to the size of a crystal.

\section{Materials and Methods}

\subsection{Photodiode}

A Photodiode is a semiconductor device generate electrical current. By converts light energy into electric current.photodiode generate current by absorbing photons. Photodiodes contains built in lenses, optical fibers, and may have small or large surface areas. A photodiode is designed to operate in reverse bias and response time are usually slower as their surface area increases. Photodiodes and regular semiconductor diode are similar except that A photodiode may be exposed to detect radiation rays photodiode are packaged with a optical fiber or window connection to allow the light energy to reach the sensitive part of the diode. A photodiode is designed to operate in reverse bias

The low-cost counter tube is similar to that of a BPW34 PIN photodiode. The plastic enclosure of the device, stops the alpha particles, without problem radiation passes through and in the diode's depletion layer it creates many electron-hole pairs. Almost, all of the 
charge carriers will be drawn away, if the diode is reverse-biased[3].It results in a small signal of current which can be processed and further amplification process is being done.If beta particles are strong enough ,they also generate a signal.The normally obtained amplitude from a counter tube is considerably larger than the amplitude of the signal produced by the photodiode and so a amplifier circuit which has a very low-noise instrumentation is needed. Another requirement the light should be completely excluded when using a photodiode as a radiation detector and as a beta[4]. Otherwise the signal we are looking for will be overwhelmed by the photocurrent. In the prototype ,a aluminium foil is mainly used.Greater number of semiconductor will be interacting with the protons. To optimise the device's sensitivity, the structure is used in a photodiode. If the sensitive area of the device is increased, there will be an increase in sensitivity. There is a decrease in its output signal, which is a disadvantage of increasing its capacitance. There is a large area and a wide intrinsic region in the commercially - available semiconductor radiation detectors. Of course simple pin photodiodes such as BPW34 are somewhat cheaper and are less sensitive than these devices. Apart from their enclosures, BPW34 photodiode are practically identical BPW34 (which is cheaper) comes in plastic package[5]. The Diode will be capable of detecting the alpha particles, when there is a possibility to remove the glass (carefully !) to expose the chip. The particles or rays must first make it through a 15Mum thick piece of aluminium (The thickness of ordinary kitchen foil). The alpha particles with energy of $4 \mathrm{MeU}$ or more will pass through, and there is no obstacle to gamma rays and beta rays. The radiation has been declared has been produced the form of flashes of light sometimes the sensor will detect the process take place when the Therefore it is impossible even for BPW34 to have some sensitivity to alpha particles[6]. It is perhaps less surprising, that a photodiode is sensitive to radiation than as before the effect has not been widely remarked. In dynamic RAMs the effect is well known, the incident radiation corrupts the stored data.Electronics is maily used to withstand radiation.

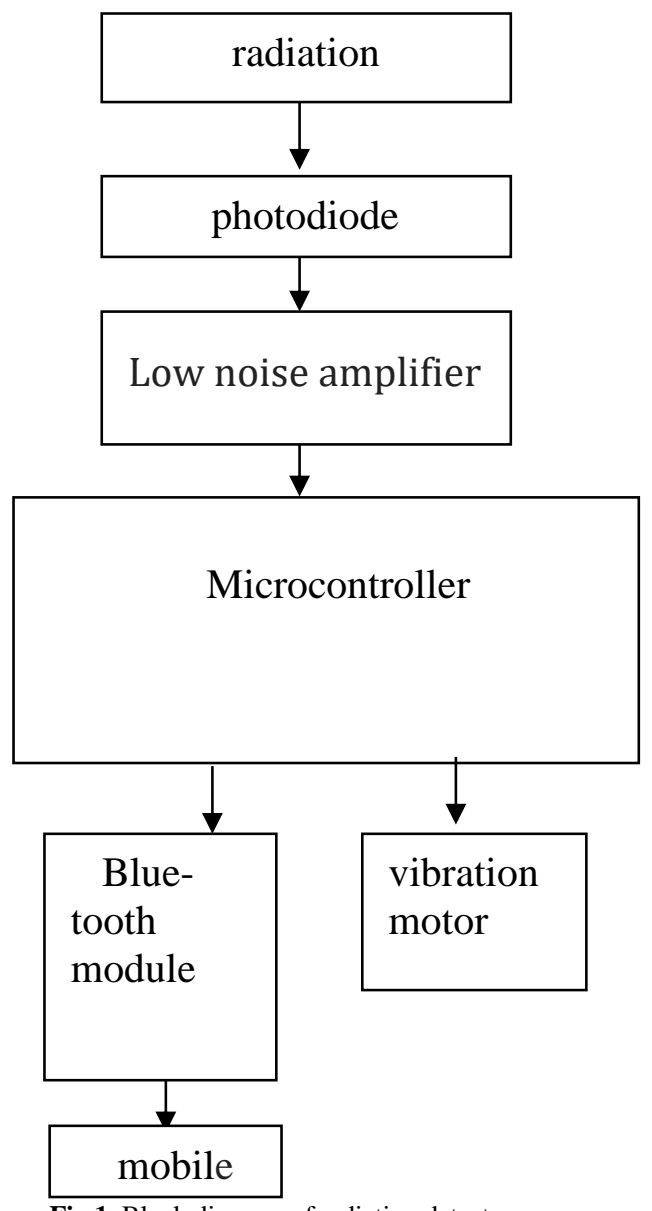

Fig.1. Block diagram of radiation detector

\subsection{Low Noise Amplifier}

It is a amplifier which will be comprising low power.It decreases the signal to noise ratio.Now the power and the noise of the input supply is increased. Low noise amplifier is commercially used in many communications.Antennas are a source of weak signals. [7]

\subsection{Microcontroller}

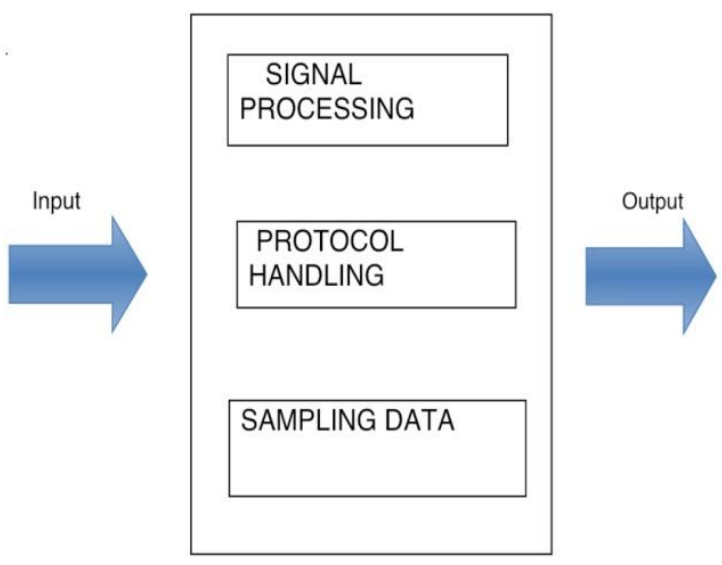

Fig.2. Microcontroller function

Micro controller are used in automated products and devices, such as engine control systems in automobile, medical implantable devices, remote controls. A micro controller is s single integrated circuit which act as a small compute it contains one or more central processing unit along with programmable input/output and memory[8].Microcontroller will give the output in two ways sudden out put as a vibration by the $3 \mathrm{v}$ vibration motor on the band and using Bluetooth module the out put signal will be transmitted to the smart phone and displayed as a digital output.

\section{Result and Discussion}

The circuit has built on a piece of breadboard and stitched in the wearable band and the photodiode has been enclosed with a black strap of rubber material to prevent light entering the sensor [9]. Then the circuit is covered and wrapped in aluminium foil and last it is grounded.It also does the role of electrical screening. The foil may cause short circuit to avoid it we just used the insulation tape[10].

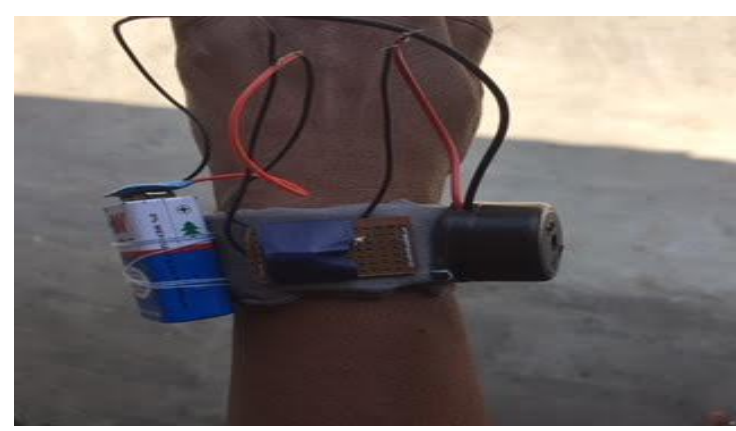

Fig.3.Schematic outcome of radiation detector

The radiation has been detector through the photodiode BPW34 and the voltage was generated from the cathode of the photodiode the 
output from the photodiode was smaller the counter tubes so we constructed the very low noise amplifier using BC549.the low noise amplifier will amplify the output from the photodiode and give the output voltage maximum voltage in this prototype we directly connected the output from the amplifier to the 2 volt buzzer which gives the out put as a sound indication in future we will a microcontroller to the amplifier output which will gives a various types of output such as vibration, LED display and by using Bluetooth module we can transmit the output directly to the smart phone.

\section{Conclusion}

This prototype is used to detect the radiation and alert the wearing person by the output sound. This wearable radiation detector detects the radiation by the photodiode BPW34 which is highly radiosensitive to avoid the photocurrent from the so the circuit and the circuit has been wrapped with the aluminium foil and grounded it will act as a screen to the photodiode helps to detect the radiation .Using microcontroller we are going to add Bluetooth module, vibration motor and LED display to give the better and accurate output details. In future we are going to reduce the size of the band and make it as a portable device and the microcontroller will be added to the circuit to perform the better output.

\section{References}

[1] Clémentine Durnez ; Vincent Goiffon ; Total Ionizing Dose Radiation-Induced Dark Current Random Telegraph Signal in Pinned Photodiode CMOS Image Sensors, IEEE Transactions on Nuclear Science ( Volume: 65, Issue: 1), 04 December 2017

[2] Derek Noble, Digicounter Link?an interface for the BBC Microcomputer and a digicounter radiation counter, Electronics Education ( Volume: 1992, Issue: 2), 1992

[3] Federico Ravotti,BPW34 Commercial p-i-n Diodes for HighLevel 1-MeV Neutron Equivalent Fluence Monitoring, IEEE Transactions on Nuclear Science, Volume: 55, Issue: 4, Aug. 2008, 10.1109/TNS.2008.2000765

[4] B.V. Grinyov; V.D. Ryzhikov; S.V. Naydenov, 07 May 2007, Radiation Detectors Scintillator-Photodiode on the Base of A2B6 Crystals for Application in Homeland Security and Medical Equipment, Nuclear Science Symposium Conference Record, 2006. IEEE , 07 May 2007, 10.1109/NSSMIC.2006.356045

[5] F. Moscatelli ; D. Passeri ; Radiation damage effects on p-type silicon detectors for high-luminosity operations: Test and modeling, Radiation and Its Effects on Components and Systems (RADECS), 2016 16th European Conference, 02 November 2017 10.1109/RADECS.2016.8093111

[6] R. N. Salikhov; A. S. Oleynik, The modern status of native thermal detectors of laser radiation, Actual Problems of Electron Devices Engineering(APEDE), 2016 International Conference, 16 March 2017, 10.1109/APEDE.2016.7878980

[7] Yury D. Mozgovoi ; Sergei A. Khritkin ; Microwave radiation of passing and counter electron beams in electrodynamics systems,

[8] vacuum Electronics Conference (IVEC), 2013 IEEE 14th International, 01 August 2013

[9] S. Yamaura; J. Nishioka ; Compact batteryless photodiode module for measurement of radiation characteristics, Antenna Measurements \& Applications (CAMA), 2017 IEEE Conference 01 February 2018 
cc) (i) Copyright $\odot 2016$ Authors. This is an open access article distributed under the Creative Commons Attribution License, which permits unre-

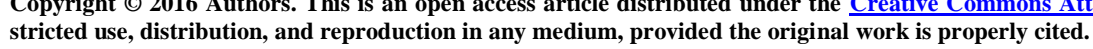

